Meta

Journal des tradlucteurs

Translators' Journal

\title{
Les technolectes dans la pratique dictionnairique générale. Quelques fragments d'une culture
}

\section{Jean-Claude Boulanger et Marie-Claude L’Homme}

Volume 36, numéro 1, mars 1991

La terminologie dans le monde : orientations et recherches

URI : https://id.erudit.org/iderudit/002113ar

DOI : https://doi.org/10.7202/002113ar

Aller au sommaire du numéro

Éditeur(s)

Les Presses de l'Université de Montréal

ISSN

0026-0452 (imprimé)

Découvrir la revue

Citer cet article

Boulanger, J.-C. \& L'Homme, M.-C. (1991). Les technolectes dans la pratique dictionnairique générale. Quelques fragments d'une culture. Meta, 36(1), 23-40. https://doi.org/10.7202/002113ar 


\title{
LES TECHNOLECTES DANS LA PRATIQUE DICTIONNAIRIQUE GÉNÉRALE. QUELQUES FRAGMENTS D'UNE CULTURE
}

\author{
Jean-Claude Boulanger et Marie-Claude L'Homme
}

Université Laval, Québec, Canada

\section{LE DICTIONNAIRE DE LANGUE, UNE RÉSIDENCE SECONDAIRE POUR LA TERMINOLOGIE}

La terminologie a maintenant atteint le stade de la maturité certaine qui en fait l'une des composantes essentielles des sciences du langage, tout spécialement lorsqu'il s'agit de mener des recherches hors de ses frontières internes comme en aménagement linguistique et en lexicographie. Elle peut prétendre au titre de discipline autonome dans le vaste champ de la linguistique parce qu'elle possède maintenant les indispensables fondements théoriques et méthodologiques qui personnalisent une science ou un art et que partout dans le monde, quelle que soit la langue cible, on lui reconnaît des assises communes et des universaux telles la notion, la démarche onomasiologique, etc. On a donc devant elle des comportements intellectuels et pragmatiques conséquents. La terminologie est devenue un corps de doctrine, de connaissance et d'expérience dont l'autonomie permet qu'on y recoure pour procéder à l'examen d'autres composantes de la linguistique, comme la lexicographie, qui sera l'objet de cette intervention.

La traditionnelle et rigide dichotomie entre la lexicographie et la terminologie, ou mieux la terminographie, n'a plus de raison de demeurer étanche. La distinction était autrefois utile afin que la terminologie puisse fonder et asseoir ses préceptes. Maintenant que cette mission est accomplie, on peut retourner au dictionnaire général (DG) et se pencher sur la perception et le traitement des technolectalismes dans cette catégorie de répertoire de mots. On sait que du strict point de vue linguistique, le dictionnaire est le lieu de rencontre privilégié de la plupart des composantes de la linguistique. $\grave{A}$ un titre ou à un autre, la phonétique, la grammaire, l'histoire de la langue, la syntaxe, la lexicologie, la sémantique, la terminologie, etc., y occupent une place prépondérante. «Les dictionnaires de langue ou dictionnaires de mots proposent des données d'ordre linguistique, c'est-à-dire : nature, genre, forme graphique et sonore du mot; significations, valeurs d'emplois, spécialisation dans les divers niveaux de langue; relations avec les autres éléments du lexique; origine et parfois histoire du mot» (Quemada 1989: 388). La consultation d'un seul article de dictionnaire atteste amplement de la grande richesse et de la variété des informations sur la langue. Les recueils de mots sont aussi des territoires privilégiés pour étudier l'histoire, la civilisation, la société et les différentes idéologies qui ont cours à un moment donné dans la vie d'une culture, d'un peuple. Ainsi le fait pour une communauté linguistique de ne pas posséder de dictionnaire national est déjà fort significatif en soi. C'est l'une des premières étapes du processus d'aménagement 
linguistique et, avec la grammaire, un préalable à l'aménagement de la terminologie, à l'aménagement de la néologie et à l'aménagement de la normalisation.

Le dictionnaire général monolingue (DGM) est un carrefour où se donnent rendezvous une somme de discours pluriels sur la société. Ces discours ont été décortiqués abondamment par les métalexicographes, les linguistes, les critiques et les évaluateurs de dictionnaires. Certains ont été davantage que d'autres la cible des observations, notamment les régionalismes, les néologismes, les emprunts et la labellisation sociale (voir Corbin/Corbin 1980 et Corbin 1980). Ce sont là, en effet, les éléments les plus immédiatement perceptibles de la norme, ceux qui atteignent directement la fibre profonde des êtres humains et qui les mettent en contact intime et immédiat avec ce que la société autorise, admet, tolère, critique, rejette, interdit ou ostracise. «Le discours lexicographique n'est pas neutre, il véhicule un contenu culturel, il émet des jugements de condamnation ou de valorisation qui s'expriment par rapport à une norme linguistique et culturelle qui prend pour référence l'univers langagier de la culture dominante» (Girardin 1987: 76).

Dans cette panoplie de discours dictionnairiques multiformes, le moins évalué est le discours sur les terminologies scientifiques et techniques. Le propos de cet article est d'amorcer l'étude de l'appareil métalinguistique utilisé pour tramer les technolectes dans les DGM.

\section{DES TERRITOIRES CONTIGUS}

L'objet de la lexicographie et celui de la terminographie présentent plusieurs similarités, d'où les difficultés d'établir des frontières bien nettes ou de trancher entre les deux. La matière commune est celle des unités lexicales qui sont recensées dans les dictionnaires et pourvues d'un traitement microstructurel adéquat fondé sur des principes dont certains sont partagés par les deux arts alors que d'autres sont révélateurs de la personnalité de chacun. Dans les dictionnaires de langue, on a l'habitude de dénommer entrée-mot une unité de traitement soumise à l'encodage métalangagier tandis que dans les dictionnaires terminologiques (DT), on désigne par entrée-notion le bloc d'unités de traitement encodées, étant entendu qu'il peut exister plusieurs unités-termes pour baptiser un seul concept. L'utilisateur décrypte des unités lexicales (signe $=$ signifiant + signifié) dans le premier cas et des termes (signe $=$ dénomination + notion) dans le second.

S'il est relativement aisé d'écarter les mots de la langue générale et les éléments de formation (affixes ou affixoïdes indigènes [-erie, contre-], savants [phon(o)-, -thèque, -ensis (ex. homo quebecensis)] ou immigrés en français [-ing, -man, -burger]) des DT, il en va tout autrement dans les DGM où les constellations de vocabulaires spécialisés sont relativement importantes et réparties sur un spectre qui va du plus visible au plus diffus. Par ailleurs, perçue sous l'angle du lexique total d'une langue, la compartimentation entre la langue quotidienne et les vocabulaires d'experts n'est pas étanche (voir le schéma dans Boulanger 1990: 4). Et cela en raison même du va-et-vient permanent entre les mots qui émigrent vers les langues de spécialité (LSP) (ex. bougie) et des vocables terminologiques qui envahissent largement la langue générale (ex. transistor, déjà vieilli, et macro-instruction, déjà réduit familièrement à macro, sans qu'il y ait risque de confusion avec son homophone masculin). À cela s'ajoute le fait qu'il existe des sphères du savoir d'expert qui chevauchent la langue usuelle et les domaines de l'expérience. De plus, si l'on se place sous l'angle de la linguistique, on ne voit pas de réelle démarcation. Les technolectes ne se dissocient pas de la langue courante quand il s'agit de la formation d'unités nouvelles. Les variations relèvent davantage de la fréquence des mécanismes convoqués, comme la syntagmatique, quelques affixes, la dérivation savante, que du moule dans lequel l'élément inédit est coulé. Ils puisent constamment dans le réservoir de 
désignations partagé et ils obéissent à la norme dominante ainsi qu'aux conventions grammaticales, morphologiques, orthographiques, syntaxiques, etc., du système général. Les LSP ont cependant toute la latitude voulue pour adapter les ressources de la LG à une situation de communication canalisée vers un domaine de la connaissance (voir Cayer 1982: 17).

Le dictionnaire de langue joue à cet égard un rôle polyvalent car il dirige ses antennes simultanément en direction du jardin de la langue usuelle et vers celui des LSP (voir L'Homme 1990 pour la discussion de ces deux notions). Or LG et LSP forment un bloc compact du point du vue du lexique cumulatif. Il est assez difficile d'estimer la part de chaque sous-lexique dans un DGM, mais quelques sondages proposent une répartition par moitié, ce qui ne semble pas très éloigné de la réalité (voir Béjoint 1988: 354 et Boulanger 1990: 16). Compte tenu de la visée du DG, à savoir capter le vocabulaire d'usage courant, on pourrait s'étonner de la consignation de si vastes amas terminologiques dans ces ouvrages. Plusieurs galaxies de mots orbitent autour d'un noyau lexical plus dense et plus apparent (ex. le groupe informatique, ordinateur, logiciel, disquette, mémoire, disque rigide; le groupe sida, HIV, LAV) alors qu'une bonne partie de la terminologie demeure inaccessible soit parce qu'elle est hors d'atteinte du télescope du lexicographe, soit parce qu'elle n'éveille pas d'intérêt. Au surplus, comme la portion du lexique général reste relativement stable d'une année sur l'autre, il faut bien admettre que dans l'avenir, les couches spécialisées du lexique fourniront de plus en plus de contingents nouveaux lorsque viendra le temps de procéder aux retouches dictionnairiques. Les ajustements macrostructurels se font de plus en plus à la hausse de même que les révisions microstructurelles qui allongent les articles (voir Boulanger 1990: 16-17). Ainsi la répartition entre les contenus de LSP et de LG tend à équilibrer la matière et à créer un rapport proportionnel qui accentue le caractère hybride des DGM. Les dictionnairistes ne font que répondre aux exigences et aux besoins de leur public respectif. Ils sont contraints de récupérer des termes spécialisés et d'en dessiner le profil dans leurs répertoires. $\mathrm{Si}$, en principe, le lexicographe ne peut pas laisser échapper un mot, il doit, en revanche, effectuer une sélection très rigoureuse dans l'ensemble des sous-lexiques technolectaux qui se répartissent sur une palette très large (alimentation, art culinaire, banque, intelligence artificielle, bioéthique, conchyliologie, etc.). Ce n'est pas le degré de spécialisation qui permet de cataloguer les termes dans un dictionnaire grand public. Tous les technolectalismes sont susceptibles de rejoindre un jour ou l'autre une macrostructure générale si le besoin est manifesté par les consommateurs (ex. microonde).

Chacune à leur manière, la lexicographie et la terminographie tendent à l'exhaustivité; mais elles procèdent en fonction de leurs méthodologies respectives. La première cherche à circonscrire explicitement ou implicitement (ex. des séries ouvertes en -able, en anti- et, naguère, en mini-) tous les mots de la langue tandis qu'une sélection rigoureuse s'impose dans les LSP (elle n'incorpore pas tous les termes en bio- ou en tomie). La seconde rassemble onomasiologiquement toutes les unités d'une sphère de l'expérience tandis qu'elle élimine tout mot outil (prépositions, articles, pronoms, etc.) et tous les morphèmes affixaux ou affixoïdaux qui ne sont pas des notions pouvant prendre place au sein d'une arborescence du savoir technique ou scientifique. Elle élimine aussi tout ce qui n'est pas conforme à l'expérience décrite; autrement dit, elle ne recrute que le vocabulaire d'une terminologie qui est univoque, «unidomaniale». Dans l'une ou l'autre situation, tout manquement aux préceptes est considéré comme une erreur méthodologique. Le programme du DGM interdit au rédacteur un traitement complet et systématique des terminologies qu'il souhaite intégrer. L'exhaustivité conceptuelle chère aux terminographes est absolument inenvisageable dans ce genre de recueil. Rares sont 
donc les terminologies complètes consignées dans un DG: seules de petites constellations pourraient prétendre à la description totale, à condition toutefois qu'elles soient en demande.

Personne ne met en doute la nécessité de la présence des technolectes dans les dictionnaires à l'usage de tous. Depuis l'origine de la lexicographie française, aucun dictionnaire de langue, petit (Dictionnaire CEC Jeunesse), moyen (Micro-Robert Plus), grand (Lexis) ou géant (Grand Robert de la langue française), monolingue, bilingue ou multilingue, ne peut s'exempter de répertorier de nombreuses terminologies. Même si en apparence, les dictionnaires courants «n'offrent pas de contenu explicitement terminologique, [ils] fournissent à la terminologie le tissu nourricier qui lui permet d'être utilisée, employée dans le discours oral et écrit, de ne pas demeurer à l'état fictif et abstrait de listes» (Rey 1985: 5). Les voies de la connaissance exigent aujourd'hui une information inflationnaire de plus en plus axée sur les expériences technologiques et scientifiques par l'entremise de la communication mondialisée et grâce à la civilisation médiatique. De plus en plus de choses à connaître rapidement, à comprendre et à interpréter entraînent une augmentation des exigences de voir représenter les savoirs technolectaux dans les dictionnaires généraux monolingues. La consignation des termes dans ces ouvrages favorise l'insertion de plus en plus évidente dans l'environnement socio-économique et elle balise l'accès à une culture technicienne et technologique qui s'accroît sans cesse selon le principe de l'offre et de la demande. À travers les mots, le dictionnaire ne fait que réunir des éléments de la culture qui est à son origine tout comme il ne peut être que le produit de cette même culture. Il est donc simultanément le révélateur linguistique d'un état de civilisation et un autoportrait d'une communauté de locuteurs. Le schéma suivant simplifie cette relation.

DICTIONNAIRE $\longrightarrow$ CULTURE
CULTURE $\longrightarrow$ DICTIONNAIRE

Lorsque la culture glisse vers l'industrialisation, la scientifisation et l'universalisation, le dictionnaire en répercute les échos tout comme il dessinait naguère le profil humaniste de la société de la Renaissance, le portrait de l'Honnête homme dixseptièmiste, la naissance et les progrès de l'univers scientifique moderne à l'époque des Lumières et le monde en devenir surgi du brassage des classes sociales à partir de la Révolution française (sur ce dernier point, voir Rey 1989b). La reconnaissance d'un français national, les débuts de son expansion territoriale au-delà des frontières européennes, l'obligation de la scolarisation, la généralisation de l'instruction, tout cela, associé au mouvement des idées, a contraint les dictionnaires à élargir leur palette d'informations afin de «répondre aux curiosités de plus en plus diversifiées d'une masse de lecteurs plus nombreuse et moins homogène» (Quemada 1989: 390). À partir du XVII e et surtout du XVIII e siècle, les répertoires devront avoir réponse à tout, aussi bien aux servitudes de la grammaire qu'aux canons esthétiques et aux désirs de connaissances encyclopédiques et spécialisées sur le monde (voir Bouverot 1986). La modernisation de la masse lexicale passe en grande partie et inévitablement par les technolectes, multiples reflets des progrès du savoir. La nature des ajouts et des mises à jour ressortit à une multiplicité de domaines, inconnus hier encore, et qui sollicitent maintenant la curiosité et l'intérêt des consulteurs. Pour chaque DG, il devient donc impérieux d'intégrer «au premier chef, de nombreux termes spéciaux des sciences et des techniques d'aujourd'hui, notamment en médecine, en informatique, biologie, sciences de l'ingénieur» (PLI 1990:5). 
La pensée contemporaine filtre inévitablement par la culture «technologicienne», Par la même occasion, les technolectalismes «ont pris une importance nouvelle du fait de la diffusion sociale des connaissances» (Rey 1989a: X). La génération naissante des dictionnaires de langue focalise son intérêt sur la science et sur la technologie, alors que la génération précédente mettait l'accent sur le développement de l'éducation et de l'information qui ouvrirait les portes à de nouveaux champs de la connaissance (voir PLI 1975: Aux lecteurs). Ayant accompli son cycle, la culture «culturelle» cède sa place à d'autres formes de culture et à des praxis innombrables.

Plusieurs indices permettent de repérer la terminologie dans les DGM. Les vocabulaires spéciaux sont présents dans n'importe quel lieu de l'article (voir Boulanger $1989 \mathrm{a}, 1989 \mathrm{~b}, 1989 \mathrm{c}, 1989 \mathrm{~d}, 1989 \mathrm{e}$ et 1990). On remarque notamment que les types particuliers de LSP s'articulent autour de deux axes principaux : celui des domaines d'activité considérés en eux-mêmes et celui des niveaux de langue, la terminologie étant alors jugée dans la microstructure par rapport à la langue courante normalisée.

Dans la suite de cet article, on s'arrêtera plus spécifiquement sur l'un de ces indices, à savoir les labels socioprofessionnels qui seront scrutés à travers la liste des abréviations (voir Boulanger 1988: 295 et suivantes). Nous concentrerons nos observations sur cet aspect et sur les discours prélexicographiques dans lesquels les programmes dictionnairiques sont détaillés. Les marques peuvent aussi être traquées dans le corps de la microstructure où elles constituent l'une des articulations majeures de l'architecture interne du discours lexicographique (voir Candel 1979 et 1983). Compte tenu de l'espace réservé à cette contribution, nous reviendrons ailleurs et plus en profondeur sur le rôle des définitions et des autres indices de domaine à l'intérieur de l'article. Nous nous contenterons également d'amorcer la réflexion sur le rapport entre la norme et les labels d'identification spécialisés. On s'intéressera ici uniquement aux descripteurs de domaine indépendamment de l'énoncé définitionnel (voir Rey-Debove 1989: 159 et suiv.). Les formules qui suivent sont exclues de l'étude :

\section{Nom que les $\mathbf{X}$ donnent à $\mathbf{Y}$}

$\mathbf{P R}$

PR

pyrite puy

PR

meuble

PR pyroscaphe

«Nom donné par Jouffroy d'Abbans au premier bateau à vapeur.»

ou

\section{Nom donné à $\mathbf{X}$}

«Nom donné à d'autres sulfures métalliques.» «Nom donné, au moyen âge, à certaines sociétés littéraires et religieuses qui organisaient des concours de poésie.»

ou

\section{Nom générique de $\mathbf{X}$}

«Nom générique des objets mobiles de formes rigides, qui concourent à l'aménagement de l'habitation, des locaux.»

ou

\section{Nom de $\mathbf{X}$}

«Nom du citrus paradisi dont les fruits viennent en grappe (grape-fruit).» 


\section{LES DISCOURS INTRODUCTIFS}

Les discours tenus sur les technolectes dans les introductions de dictionnaires portent essentiellement sur deux aspects: le premier concerne le choix des mots ou des sens spéciaux, terminologiques, techniques, scientifiques ou didactiques, à retenir dans le dictionnaire. Le second aspect touche le protocole d'encodage des unités sélectionnées.

Les préfaciers ou les rédacteurs des discours commerciaux peuvent discuter le problème des terminologies sous deux angles. S'il s'agit d'un dictionnaire qui en est à sa première édition on stipulera volontiers que l'introduction des vocables scientifiques et techniques est indispensable, «qu'aucun domaine de la connaissance n'a été négligé» (Lexis 1975 : VII), que les «terminologies spécialisées occupent une place de plus en plus importante dans les milieux de travail, dans les media [sic] et dans les communications quotidiennes, [qu'on] les a examinées avec beaucoup d'attention afin d'en extraire tous les éléments nécessaires à une meilleure compréhension du monde contemporain» (Poirier 1988: XVI). Dans le cas des ouvrages qui en sont à une nouvelle mouture (réédition ou refonte) on justifie l'élargissement des cadres de la nomenclature par des ajouts de néologismes formels ou sémantiques et par l'addition de régionalismes; mais c'est surtout l'introduction de termes représentatifs de centres d'intérêt très variés qui a pris de l'importance au cours des récentes années. «Plusieurs milliers de néologismes et de termes spéciaux récemment diffusés s'y [aux 50000 entrées de la précédente édition] ajoutent dans la présente édition, ainsi que des acceptions et des expressions nouvelles. Ce sont les vocabulaires scientifiques et techniques qui se taillent la part du lion» (Rey 1985: XVII-XVIII, italiques ajoutées). Cependant, dans l'une et l'autre situations, les contenus sont toujours orientés vers l'obligation d'offrir au public un produit qui soit à la fine pointe de la modernité socioscientifique et sociotechnologique. «En ce qui concerne les vocabulaires scientifiques, on a fait une large place aux vocabulaires des sciences et des techniques, et souvent à des termes d'un haut degré de technicité, dans tous les domaines qui occupent une place essentielle dans la civilisation contemporaine: économie politique, informatique, écologie, électronique, industrie du pétrole, sciences humaines, biologie et médecine. On s'est, en revanche, limité à l'essentiel pour les activités ou les techniques qui sont des survivances ou n'intéressent que des groupes restreints (héraldique, vénerie, bourrellerie, par exemple)» (Lexis 1979: VI).

Quel que soit le répertoire consulté et à condition qu'il soit pourvu d'une introduction tant soit peu étoffée, tous les dictionnairistes insistent sur le fait qu'il est impensable aujourd'hui de constituer une nomenclature de dictionnaire de langue sans y incorporer des éléments techno-scientifiques (voir Girardin 1987: 81). Certes, tous endossent l'idée que le DGM n'est pas le lieu pour recenser systématiquement tous les vocables des multiples expériences humaines. Une sélection stricte s'impose. Parmi l'ensemble des termes disponibles, on distingue les unités qui permettent de décrire le fonctionnement de la science ou de la technique, celles qui appartiennent à des séries ou à des énumérations ouvertes et celles qui forment des grappes volumineuses et serrées : «on ne pouvait raisonnablement envisager, dans le cadre d'un seul volume, de recenser, en botanique et en zoologie, les innombrables animaux et plantes exotiques, en géologie les noms de tous les minéraux roches et fossiles» (Lexis 1979: VII). Ce qui intéresse le destinataire, c'est l'essentiel ou les éléments de ces terminologies susceptibles de surgir au détour d'une lecture, d'une interrogation, d'une recherche dont l'objectif premier n'est pas d'ordre conceptuel ou systématique, au sens où l'entendent les experts d'un secteur d'activité. Ces termes-mots sont choisis en fonction d'un environnement socioculturel qui rend leur description lexicographique obligatoire.

Le principal critère de sélection généralement avancé est le contact que les usagers ordinaires ónt avec ces unités et les besoins qu'ils ont de pouvoir décoder le terme sans 
avoir à recourir à un dictionnaire terminologique ou à un dérivé informatisé. Somme toute, le sort réservé à un terme (inclusion ou exclusion) est l'intérêt qu'il suscite pour le grand public, l'impact lexical, et non pas notionnel, qu'il a sur la langue usuelle d'un groupe de locuteurs donné, en un espace communautaire donné. Mais quel que soit l'ordre de grandeur de la nomenclature, un tamisage fin du lexique spécialisé est indispensable.

La majorité des dictionnaires ne précise pas vraiment quelle est la provenance des contingents technolectaux. Quelques-uns citent des sources premières, par exemple, le DFP : XVI, qui a puisé et filtré une partie des termes consignés dans les publications terminographiques d'un organisme officiel, l'Office de la langue française du Québec; d'autres, comme le PR et le GRLF, mentionnent la consultation de spécialistes, par exemple des informaticiens, des automaticiens, des biologistes, des médecins, pour évaluer les terminologies idoines; d'autres encore puisent dans leurs fonds encyclopédiques constitués au fil de leur histoire, ainsi qu'on le fait dans les ouvrages de la maison Larousse. Dans la réalité, la plupart des répertoires recourent simultanément aux différentes possibilités: dictionnaires techno-scientifiques, experts, fonds d'archives. En outre, le lexicographe cite directement des extraits d'ouvrages, de collectifs ou de revues spécialisés; la pratique est méthodologiquement standardisée depuis une dizaine d'années, même si on y recourait occasionnellement depuis longtemps. D'abord sporadique, la méthode tend à se répandre de plus en plus (voir Rey 1985: XXXVIII et le Lexis). Auparavant, l'illustration contextuelle d'un terme provenait surtout de la littérature produite par les grands auteurs:

PR oxygène

\begin{tabular}{|c|c|c|}
\hline \multirow{5}{*}{ Lexis } & lophophore & $\begin{array}{l}\text { «Son grand chapeau de paille noire... avec une } \\
\text { fantaisie de lophophore.» (Aragon) }\end{array}$ \\
\hline & équin & $\begin{array}{l}\text { «Cet équin, large en effet comme un pied de cheval.» } \\
\text { (Flaubert) }\end{array}$ \\
\hline & ove & $\begin{array}{l}\text { «Une frise sculptée qui, de loin, m'avait semblé faite } \\
\text { de fleurs ou d'oves.» (Butor) }\end{array}$ \\
\hline & ventricule & $\begin{array}{l}\text { «Une tumeur épithéliale kystique développée aux } \\
\text { dépends du revêtement du troisième ventricule.» } \\
\text { (Cendrars) }\end{array}$ \\
\hline & sanie & $\begin{array}{l}\text { «Les mouvements du gorille brassaient de lourdes } \\
\text { odeurs de crasse et de sanie.» (Aymé) }\end{array}$ \\
\hline
\end{tabular}

Le second aspect des discours commerciaux concerne les modalités de marquage, c'est-à-dire les critères sous-jacents à la pose des balises métalinguistiques. Là aussi quelques règles orientent les décisions. De manière absolue, on procède à une labellisation des formes qui se détachent de l'emploi général, banal, courant du mot, au sens où l'entendent les lexicographes. La marque, comme son nom le laisse deviner, isole et signale tout ce qui va au-delà ou qui se fige en-deçà du langage témoin qu'on décrit comme la langue et qui gouverne l'usage (voir Rey-Debove 1980: 40). Donc, en principe, tous les termes devraient être marqués - il serait d'ailleurs préférable de dire tous les sens puisque le descripteur précède la définition même dans les articles monosémiques. La démarche est purement mathématique: l'élément qui relève de la norme neutralisante n'est accompagné d'aucune étiquette, c'est-à-dire qu'il n'est pas considéré comme relevant d'un niveau techno-scientifique, tandis que tout ce qui 
s'oppose à cette neutralité lexicale, autrement dit les LSP, devrait par conséquent être hiérarchisé par rapport à ce point d'équilibre qu'est la norme, miroir de la langue usuelle.

\section{LES NORMES SOCIOPROFESSIONNELLES}

La réalité de l'observation démontre qu'il n'en va pas ainsi. Le terme ne sera escorté d'un code socioprofessionnel que s'il est mal connu dans le grand public ou considéré comme tel par le lexicographe. Ainsi, écologiste, écomusée ne sont pas marqués dans le PR, le DFP et le PLI 1990 alors que binaire est encodé à plusieurs paliers : PR : Arithm., Chim., Mus.; DFP : Chim., Math., Mus.; PLI 1990 : Math., Chim., Mus., Astron. Dans le Lexis, binaire est noté successivement: Math., Mus., Chim., Inform., Ling., tandis que dans le GRLF, la structure présente la configuration sémantique suivante:

\begin{tabular}{|c|c|}
\hline $\begin{array}{l}\text { a. } \\
\text { b. } \\
\text { c. } \\
\text { c. } \\
4 .\end{array}$ & $\begin{array}{l}\text { Arithm. vx. } \\
\text { Mod. } \\
\text { Inform. } \\
\text { Log. } \\
\text { Chim. } \\
\text { Astron. } \\
\text { Mus. }\end{array}$ \\
\hline
\end{tabular}

L'absence d'un motif technolectal dénote en premier lieu la diffusion de l'unité lexicale dans la langue générale, sa métamorphose du statut de terme à celui de mot courant (voir humidificateur marqué tech. dans le PR et le DFP, non marqué dans le Lexis et le PLI 1990; cémentation non marqué dans le PR, marqué métall. dans le DFP et le PLI 1990, géol., métall. et chim. dans le Lexis; écliptique non marqué dans le PR et le DFP, marqué astron. dans le PLI 1990 et le Lexis. Le jugement subjectif du lexicographe doit ici être pris en considération, même si une grande partie du contenu du dictionnaire est héritée de ses devanciers et que le rédacteur est considéré comme le porte-parole d'une communauté.

«Le lecteur ne doit jamais oublier que les abréviations concernent l'usage linguistique et lui seul; qu'elles ne doivent pas figurer devant un mot courant, même si ce mot désigne un objet scientifique : écologie, transistor, électronique appartiennent, bien ou mal compris, au français de M. Tout-le-Monde» (Rey 1985: XLI). L'argumentation. repose également ici sur le constat que les métaphores techniques augmentent sans cesse et qu'elles colorent de plus en plus fréquemment la langue quotidienne. Beaucoup d'entre elles sont couramment utilisées sans être toujours bien appréhendées par les locuteurs. Mais au premier chef, lorsque le lexicographe épingle une unité, c'est la forme linguistique qu'il pointe et non pas le référent extralinguistique ou le concept: «[...] c'est toujours le mot, la forme linguistique, et non pas la notion ou la chose qui est qualifiée» (Rey 1985: XLI). Le label restreint l'emploi normal du mot à un domaine précis. Mais en réalité, un mot ne peut être marqué en soi. Il revêt toujours une valeur de connotation dans les circonstances où on l'utilise, ces circonstances étant ici le secteur d'activités professionnelles. Le descripteur peut aussi être perçu comme un indice de renforcement de la norme professionnelle et/ou courante. Le consulteur est alors appelé à résoudre une formule semblable à celle-ci : 
le mot $\mathbf{X}$ caractérise un discours appartenant au technolecte $\mathbf{Y}$ : - le mot parallaxe caractérise un discours appartenant à l'astronomie

- le mot vernal caractérise un discours appartenant à l'astronomie

La marque possède la vertu de ranger immédiatement la définition du mot dans un macrocontexte de connaissance jamais isolé puisque l'article est construit suivant des principes sémasiologiques (voir binaire ci-dessus). Elle a aussi la propriété (qualité ou défaut?) de la limiter à cette situation de communication même s'il est évident que l'énoncé peut aussi convenir à d'autres champs du savoir. En revanche, plusieurs mots d'un même domaine étant disséminés dans le répertoire, l'abréviation joue le rôle d'une tête chercheuse notionnelle. Ce qui n'exclut pas la liberté d'estampiller ou non les unités lexicales d'un seul domaine ou de varier les étiquettes (PR: ordinateur et programmeur: non marqués, programme et logiciel: Techn., disquette et matériel: Inform.).

Par ailleurs, il est clairement stipulé dans les dictionnaires que «l'absence de ces abréviations devant un mot ou un sens signifie que ce mot, ce sens, sont d'emploi normal pour une personne cultivée non spécialiste, même s'ils appartiennent à un domaine spécialisé» (Rey 1989a: XVII). Mais ici, comme le souligne Rey, des éléments subjectifs entrent en jeu afin de savoir ce qui est spécialisé ou courant pour un consulteur. Le DG a comme premier objectif de circonscrire la nature linguistique de l'unité lexicale. Ce n'est que par projection secondaire et par extrapolation que le rapport avec l'objet de l'univers, le référent extralinguistique est déduit. Dans le DG, la marque technoprofessionnelle ne reflète que la seule norme linguistique sociale et le seul usage social banalisé ou considéré comme tel. Elle n'est pas fondée sur la base de l'expérience individuelle ou groupale; elle émerge du jugement que porte le lexicographe sur la norme sociale dont les limites correspondent au comportement moyen du public consommateur. Pour asseoir son évaluation de la norme admissible, il consulte des dictionnaires portant sur l'argot, les régionalismes, les néologismes, les terminologies, etc. La pratique du marquage socioprofessionnel suivra donc le même cheminement qui reflète une norme prévalante et les préjugés de la culture dominante. «Les marques ont pour objet d'expliciter des écarts par rapport à un usage contemporain neutre qui ne peut être que théorique et qui suppose donc la construction d'un modèle normatif (ce qui ne veut pas dire prescriptif)» (Girardin 1987: 92).

Le DGM accorde donc plus d'importance à la généralisation ou à la banalisation d'un usage spécialisé qu'à sa classification dans un ensemble notionnel où il fonctionne dans une série d'éléments interdépendants. Les labels professionnels ne sont pas notés pour justifier une organisation systémique de tous les mots de la nomenclature qui revendiquent le même signal puisque le DGM n'a pas d'objectif de structuration conceptuelle arborescente quel que soit le domaine considéré. En terminographie, le regroupement notionnel et le dégroupement homonymique sont méthodologiquement fondamentaux. Les indicatifs permettent tout au plus de glisser de la langue à la thématique, des connaissances linguistiques vers un savoir quasi encyclopédique en effleurant au passage l'indispensable notion des terminologues sans entrer cependant dans ses particularités onomasiologiques et dans ses dimensions logiques. Le DG reste sous le seuil de la typologie classologique. En cela, le lexicographe respecte la ligne de conduite dessinée pour le programme du dictionnaire confectionné.

En outre, un terme répertorié dans un dictionnaire courant, est réputé se diffuser dans la langue usuelle: ainsi informatique, intelligence artificielle, macrobiotique, télex dans le PLI 1990 et le PR. Il se lexicalise, contrairement au phénomène de la terminologisation dans les DT. Cette lexicalisation s'accompagne souvent d'une 
modification, d'une atténuation, d'une dérivation ou d'une perte sur le plan sémique ou sémantique. Cette perte ou cette réinterprétation des caractères des logiciens, dont la série complète est obligatoire pour décrire adéquatement une notion, est plus ou moins compensée par l'attribution d'un descripteur abrégé notant l'appartenance du terme à une LSP. Le passage du terme à l'état de mot (LSP $\rightarrow$ LG) s'effectue généralement par la focalisation sur des traits fonctionnels mais surtout sur des traits sémantiques. Certains sèmes compositionnels demeurent trop spécialisés pour quitter l'orbite de leur LSP et rejoindre les colonnes d'un DGM, sauf bien entendu pour des taxinomies, des nomenclatures chimiques ou quelques autres systèmes fermés ou assimilés. Voici l'exemple d'un terme vérifié dans deux dictionnaires de langue et dans deux dictionnaires terminologiques et pour lequel les deux définitions généralisées n'ont apparemment pas de traits communs lorsqu'on les compare:

\section{hétéronyme}

PR «Ling. Qui n'a pas le même nom.»

Lexis «Ling. Équivalent d'un mot dans une autre langue.»

DdL $\quad \ll[. .$.$] on appelle hétéronymes les mots de racine différente, mais$ formant ensemble une structure sémantique.»

DDL «Dans une langue : mots dont les signifiés sont en rapport d'intersection (qui ont donc un ou plusieurs éléments de sens communs), mais qu'on ne peut classer ni parmi les synonymes, ni parmi les antonymes.

$[\ldots]$

D'une langue à une autre: mots de sens voisin — mais non identique

- considérés plus ou moins équivalents, donc employés parfois

en traduction.»

\section{CLÉS POUR LE CODE ABRÉVIATIF}

La taxinomie des faits et des usages technolectaux est ciselée autour d'une série d'empreintes dont chaque dictionnaire doit fournir les clés dans une liste d'abréviations résolues et explicitées à la convenance de ses lecteurs. Les dictionnaires fournissent habituellement une liste relativement complète des signes conventionnels et des abréviations utilisées. Celles-ci renvoient aux marques métalinguistiques, professionnelles, géographiques, historiques, etc. Les listes figurent à la suite de la présentation et avant le dictionnaire alphabétique.

Le catalogue général inventorie les différentes catégories d'abréviations utilisées dans les articles. Celles-ci renvoient à des marques de divers ordres comme celles du tableau suivant (les variantes de caractères sont respectées). 
Tableau 1 : Échantillon de catégories d'abréviations

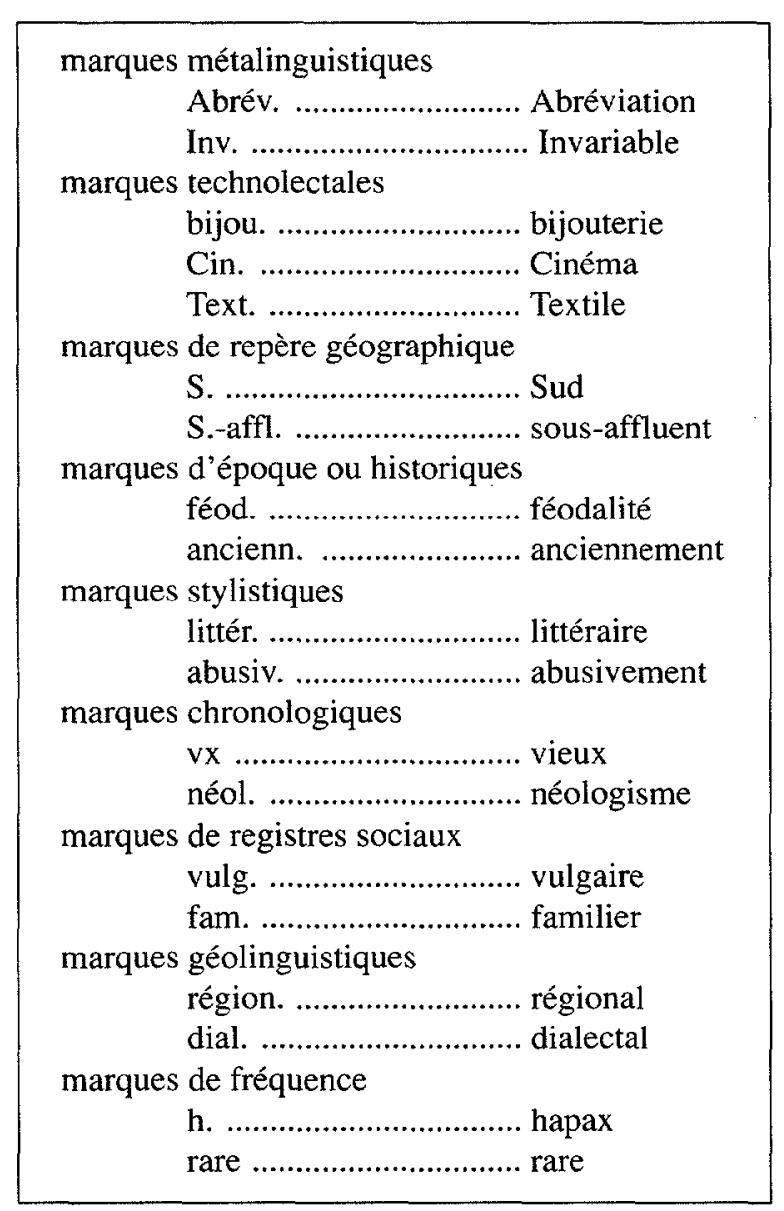

Les étiquettes technolectales peuvent renvoyer à:

$\begin{array}{llll}\text { - une science } & \rightarrow & \text { géol. } & \text { géologie } \\ \text { - une technique } & \rightarrow & \text { typogr. } & \text { typographie } \\ \text { - une technologie } & \rightarrow & \text { inform. } & \text { informatique } \\ \text { - une science humaine } & \longrightarrow & \text { litt. } & \text { littérature } \\ \text { - un art } & \rightarrow & \text { jard. } & \text { art des jardins } \\ \text { - un métier } & \longrightarrow & \text { menuis. } & \text { menuiserie } \\ \text { - une profession } & \rightarrow & \text { chim. } & \text { chimiste } \\ \text { - etc. } & & & \end{array}$

Tous les dictionnaires témoins fusionnent les différentes marques dans un seul registre; l'unique exception est le DFP qui propose trois listes distinctes: une liste générale regroupant les «Abréviations», une liste recensant les marques métalinguistiques, les «Rubriques de langue», et une liste rassemblant les descripteurs 
scientifiques et techniques, les «Indications de vocabulaire de spécialité». Il est quelquefois ardu de distinguer les motifs de domaine des autres marques à l'intérieur de ces listes mais dans la plupart des cas, les lexicographes ont recours à certains procédés de mise en évidence afin de faire ressortir les distinctions entre les différents groupes de marques. Dans le PR, sauf de rares exceptions (AFNOR, ANT., HOM., PROV., REM., V.), toutes les abréviations de la liste sont en italiques minuscules et suivies de paraphrases explicatives qui en facilitent le décodage:

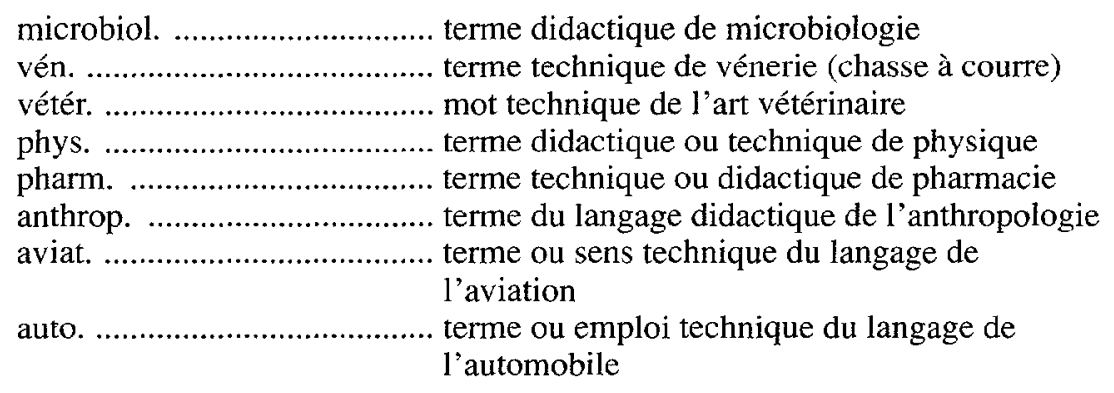

Dans le PLI 1990, les abréviations de domaines sont en petites capitales sans autre commentaire explicatif que leur résolution en toutes lettres:

AGRIC. agriculture

ALCH. alchimie

ALG. algèbre

Cependant, lorsque le domaine n'est pas abrégé, la formule est la suivante:

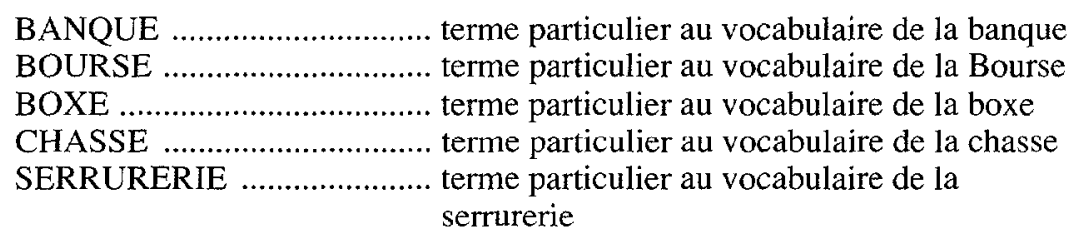

On remarque une exception à la règle:

CONSTR. terme technique de la construction

Dans le DFP, les marques de LSP apparaissent dans deux des trois listes sans que le protocole de décodage soit expliqué. Des abréviations semblables ou différentes renvoyant au même domaine sont fréquentes et il n'y a pas de réciprocité parfaite entre les deux listes partiellement récurrentes (voir le tableau 2 ). 
Tableau 2: Les groupes de marques du DFP

\begin{tabular}{|c|c|}
\hline Abréviations & Indications de vocabulaire... \\
\hline 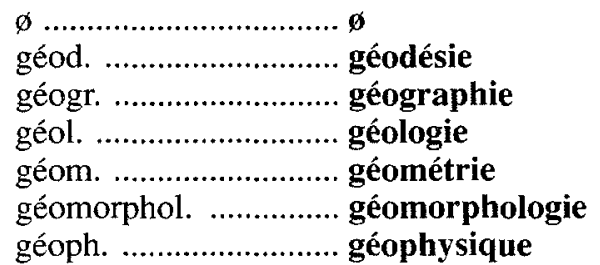 & 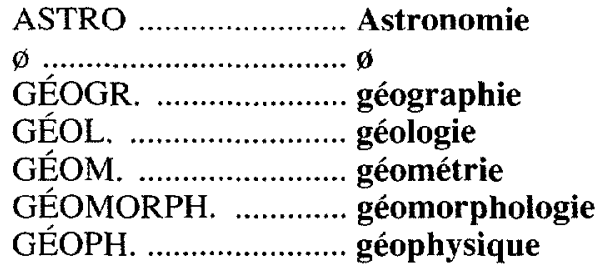 \\
\hline
\end{tabular}

Dans la première liste, les abréviations sont données en lettres minuscules et résolues en caractères gras; dans l'autre liste, elles apparaissent en petites capitales et une majuscule sert d'amorce à la résolution en gras. Deux codes abréviatifs différents sont utilisés pour le domaine de la géomorphologie.

Les deux éditions du Lexis n'utilisent aucun procédé même typographique pour distinguer les marques technolectales des autres étiquettes. Elles sont toutes données en italiques :

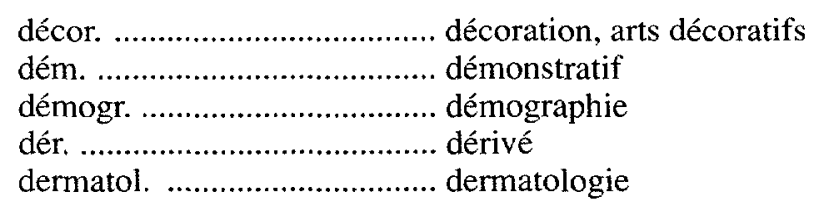

Quelques abréviations ont une double interprétation:

PR

méd.

$\rightarrow 1^{\circ}$ terme didactique de médecine $[\ldots]$

$2^{\circ}$ médical (lat. méd., lang. méd.)

PLI $1990 \quad$ cuis. $\quad \rightarrow$ cuisine, art culinaire

parfois même une triple interprétation :

DFP dém. $\rightarrow$ démonstratif, démocratie, démocratique

tandis que deux abréviations différentes renvoient parfois à un seul domaine :

PR myth., mythol. $\rightarrow$ terme didactique de mythologie

L'importance numérique des marques domaniales n'est pas à négliger dans les DGM (voir le tableau 3). 
Tableau 3: Importance numérique des marques

\begin{tabular}{|l|c|c|}
\hline \multicolumn{3}{|c|}{ Nombre de marques dans différents dictionnaires } \\
(lettres A-D) \\
\hline Dictionnaires & Techno-marques & Autres marques \\
\hline PR & 65 & 67 \\
PLI 1990 & 75 & 50 \\
DFP & 69 & 101 \\
Lexis 1979 & 93 & 152 \\
\hline
\end{tabular}

La somme des estampilles technolectales dépasse largement celle de chaque autre groupe pris individuellement; les résultats rassemblés sont cumulatifs pour la séquence de lettres étudiée.

L'analyse des tableaux des marques révèle aussi comment les dictionnaires s'enrichissent lors des mises à jour (nouvelle édition ou révision). Sans permettre une évaluation quantitative des nouvelles acquisitions, ils renseignent néanmoins sur l'arrivée de domaines inédits et sur l'usure ou sur la disparition d'autres secteurs artisanaux, historiques ou passés de mode (ex. DHLF : baronnie, GRLF : bonneterie, cosmographie). L'augmentation du nombre de domaines dans lesquels on a puisé des termes qui enrichissent les nomenclatures témoigne de la diffusion des terminologies dans le monde moderne et de leur spécialisation de plus en plus poussée. Dans chaque couple de dictionnaires présentés dans le tableau 4 , les statistiques sont éloquentes.

Tableau 4 : Rapport statistique sur les marques

\begin{tabular}{|c|c|c|c|c|c|}
\hline \multicolumn{7}{|c|}{ Marques apparues ou disparues (lettres A-D) } \\
\hline Dictionnaires & Années & Marques & Ajouts & Retraits & $\begin{array}{c}\text { Nomenclatures } \\
\text { totales }\end{array}$ \\
\hline DHLF & 1980 & 72 & $\emptyset$ & $\emptyset$ & 50000 \\
DFP & 1988 & 69 & 0 & 3 & 62000 \\
\hline PR & 1967 & 51 & $\emptyset$ & $\emptyset$ & 50000 \\
PR & 1989 & 65 & 14 & 0 & 58000 \\
\hline PLI & 1975 & 42 & $\emptyset$ & $\emptyset$ & 45000 \\
PLI & 1990 & 75 & 36 & 3 & 58000 \\
\hline Lexis & 1975 & 93 & $\emptyset$ & $\emptyset$ & 70000 \\
Lexis & 1979 & 93 & 0 & 0 & 76000 \\
\hline GRLF & 1951 & 66 & $\emptyset$ & $\emptyset$ & 58000 \\
GRLF & 1985 & 80 & 35 & 21 & 80000 \\
\hline \multicolumn{6}{|c|}{$\emptyset=>$ ne s'applique pas pour l'édition plus ancienne } \\
\hline
\end{tabular}


Dans certains cas, on a ajouté des descripteurs qui ne faisaient pas l'objet d'un intérêt particulier lors d'éditions antérieures. Par exemple, l'édition de 1989 du PR (en réalité une somme de tirages augmentés de la seconde édition de 1977) a intégré à sa nomenclature des termes provenant des domaines de l'informatique, de l'alpinisme, de la cybernétique. L'édition du PLI 1990 répertorie des termes des domaines de l'acoustique, de l'administration, de l'aviculture, de l'industrie du pétrole. Les lexicographes ont aussi procédé à une fragmentation de quelques domaines correspondant à une spécialisation du savoir. Par exemple, dans l'édition de 1990 du PLI, on retrouve les domaines de la chimie et la chimie organique alors que l'édition de 1975 considérait un seul grand domaine pour la chimie. En 1990, le PLI recense des termes de l'art contemporain, des beaux-arts, des arts décoratifs, des arts graphiques et des arts plastiques alors qu'en 1975 seuls les beaux-arts et les arts graphiques étaient représentés. La technique du classement des descripteurs sous une forme arborescente n'est cependant pas nouvelle. Les huit dictionnaires du tableau présentent ce genre de classement en réseau pour le droit. L'augmentation des domaines est aussi liée à l'accroissement des nomenclatures d'une édition à l'autre. La majeure partie des entrées nouvelles proviennent des technolectes (voir Boulanger 1990: 16-17). Mais, existe-t-il toujours une corrélation entre l'addition d'un descripteur à la liste et l'introduction de termes s'y raccrochant? Des termes d'algèbre et d'arithmétique étaient définis dans les PLI bien avant qu'on songe à les pourvoir d'un feu de signalisation.

\section{CONCLUSION}

Les marques socioprofessionnelles ne s'opposent pas vraiment aux autres catégories de labels: chronologiques, géographiques, stylistiques, registres de langue sociaux, etc. Dans la tradition lexicographique française, ces dernières sont toutes prescriptives alors que les premières ont des visées descriptives et qu'elles servent à connoter l'emploi d'un mot dans une sphère d'activité. Au même titre que les autres catégories, elles font cependant partie du catalogue du métalangage dictionnairique.

«De toutes façons, l'utilisateur a toujours tendance à interpréter de façon prescriptive les informations données par le dictionnaire, quel que soit l'esprit dans lequel elles ont été conçues, ce qui explique la violence des réactions au sujet des marques d'usage» (Béjoint 1981: 72). Mais les marques spéciales sont-elles si restrictives et normalisantes, dans le sens de «bon et de bel usage»? Sont-elles uniquement un simple appareil hiérarchisant des emplois qui cheminent du plus général au plus spécifique qu'on peut appeler un sous-code situationnel (voir Béjoint 1981:72), qui varie non pas avec les personnes mais en raison des circonstances de communication, la situation étant ici le sujet ou le domaine? Si l'on considère que le rôle du dictionnaire consiste à identifier tout ce qui dévie par comparaison avec un code normatif sélectionné plus ou moins arbitrairement, alors la réponse plaide clairement en faveur de la prescription. «Les marques d'usage, même si elles sont descriptives, le sont d'un écart par rapport à une langue neutre, normale, que le lexicographe a choisie comme modèle de description, et la description se rapproche ainsi de la prescription» (Béjoint 1981: 73). À la restriction près, toutefois, que parmi l'éventail des groupes de marques possibles, seul l'ensemble des descripteurs relatifs aux réseaux des activités de l'expérience technique ou scientifique met la langue en relation avec le référentiel.

Contrairement aux autres catégories de marques, les secteurs couverts par les labels technolectaux sont individualisants et en principe non redondants, ce qui évite les recoupements. Chaque étiquette de domaine correspond à une seule case précise dans la configuration des champs du savoir. L'introduction d'une abréviation de domaine devant l'énoncé définitionnel active ce seul sémantisme et immobilise les autres énoncés codés 
ou non. Fort peu de codes sont englobants ou génériques; ceux qui existent renvoient le lecteur à une sphère ouverte, comme didact., sc. (PR: «terme didactique du langage scientifique et appartenant au domaine de plusieurs sciences»), techn. (voir Corbin/Corbin 1980 : 247). Ainsi lorsque 1'unité binaire est cataloguée tour à tour dans les domaines de l'arithmétique, des mathématiques, de la logique, de l'informatique, de la chimie, de l'astronomie, de la linguistique et de la musique, le dictionnairiste reconnaît qu'il existe dans le DGM une sorte d'homonymie terminologique par référence aux systèmes onomasiologiques fermés des LSP. La polysémie microstructurelle des ouvrages de langue est due à la méthode sémasiologique qui oblige à fournir la plupart des sens d'une forme linguistique sélectionnée comme entrée. Cependant, la polysémisation à tendance homonymique n'est pas systématique. Ainsi l'interprétation sémantique d'un mot comme bureau échappe totalement à l'intersection avec l'homonymisation. Cette formule lexicographique n'est pas technolectalisée dans les dictionnaires courants : aucun des huit sens du PR, des sept sens du DFP, des sept sens du PLI 1990 ou des treize sens du GRLF ne sont porteurs de descripteurs même si certaines affectations sémantiques de ce mot collent à des réalités nettement professionnelles. Les grandes divisions internes des articles constituent les seuls indices de la spécialisation des significations. Bien d'autres catégories de mots ont en apparence un statut de passe-partout sémantique; ils échappent ainsi au catalogage en raison de leur identification quasi automatique à la couche du vocabulaire général; ils ne sont pas munis de motifs professionnels même si parfois un ou des scns relèvent nettement d'une série de concepts organisés. C'est le cas de plusieurs verbes faussement confinés à la polysémie ordinaire provignante: PR : faire (39 sens), prendre (43 sens), mettre ( 37 sens, sauf un qui est marqué mar.), passer (40 sens, sauf un qui est marqué comm.) (voir Loffler-Laurian 1982 et 1983). À ce niveau, il ne paraît pas absurde ou inconvenant de penser qu'une étude approfondie de l'usage des termes, de leur transformation de l'état de support linguistique des notions à celui de l'autoreprésentation lexicale, du passage d'un savoir spécial implicite à un savoir linguistique explicite par l'intermédiaire du dictionnaire, qu'une étude de ce genre permettrait une approche fructueuse de l'art et de la praxis de la lexicographie (voir Handwerker 1989: 85).

Comme on le perçoit à la lumière des quelques fragments de l'organisation des technolectes à travers les dictionnaires généraux monolingues, les terminologies ne font pas l'objet d'un traitement en soi. Ce qui importe d'abord, c'est de capturer une unité lexicale spécialisée et de tenir sur elle un discours microstructurel dont l'enveloppe est normative et assimilée à la langue usuelle des locuteurs. Il s'agit d'une sorte de rite de passage du savoir d'expert au savoir lexical, le dictionnaire de langue ne se prononçant que sur des mots. La marque lexicographie partiellement un terme, qui représente un concept dans le monde terminographique, c'est-à-dire qu'elle le métamorphose en mot. On passe alors de l'axe du savoir sur le monde à l'axe du savoir sur le linguistique. Une forme est normale, autrement dit dépourvue de marque, ou opposée à différenciée, c'està-dire marquée. L'énoncé que contient une rubrique est inscrit dans une relation duelle en ce sens que quelque chose de standard s'oppose à quelque chose qui n'est pas standard. En apparence, le discours se veut moins prescriptif en ce qui regarde les technolectalismes puisque ceux-ci ne sous-entendent pas l'existence de formes opposées plus acceptables. Il en va également ainsi pour les régionalismes qui réfèrent à des éléments conceptuels spécifiques d'un territoire. Mais la menace de la norme plane toujours au-dessus du réseau des indices réducteurs (voir Boulanger 1989f et 1989g). Dans le dictionnaire de langue, le système des marques des domaines conceptualisés n'est qu'une suite d'indices classologiques assimilés à des niveaux de langue 
socioprofessionnels qui ne représentent qu'une autre bande du spectre qu'est une norme dictionnairique.

\section{BIBLIOGRAPHIE}

\section{Linguistique}

BÉJOINT, Henri (1981): «Les marques d'usage dans les dictionnaires de langue anglaise», Tréma, Université de Paris VII (Sorbonne Nouvelle), 7, pp. 69-74. [La langue aux États-Unis].

BÉJOINT, Henri (1988): «Scientific and Technical Words in General Dictionaries», International Journal of Lexicography, 1-4, hiver, pp. 354-368.

BOULANGER, Jean-Claude (1988): «Les dictionnaires et la néologie: le point de vue du consommateur», Actes du Colloque Terminologie et technologies nouvelles, Paris-La Défense, 9 au 11 décembre 1985, Montréal, Office de la langue française, Gouvernement du Québec, pp. 291-318.

BOULANGER, Jean-Claude (1989a): «Le statut du syntagme dans les dictionnaires généraux monolingues», Meta, 34-3, pp. 360-369.

BOULANGER, Jean-Claude (1989b) : «La place du syntagme dans le dictionnaire de langue», Meta, 34-3, pp. 516-528.

BOULANGER, Jean-Claude (1989c): «La cartographie lexicographique des avis officiels», Actes du Colloque La description des langues naturelles en vue d'applications linguistiques, Université Laval, Québec, 7-9 décembre 1988, coll. «K», n 10, Québec, Centre international de recherche sur le bilinguisme, Université Laval, pp. 39-52.

BOULANGER, Jean-Claude (1989d): «Lexicographie et politique langagière: l'exemple français des avis officiels», Dictionnaires. Encyclopédie internationale de lexicographie, Éditée par Franz Josef Hausmann, Oskar Reichmann, Herbert Ernst Wiegand et Ladislav Zgusta, tome 1, Berlin-New York, Walter de Gruyter, pp. 46-62.

BOULANGER, Jean-Claude (1989e) : «Le traitement des terminologies dans les dictionnaires de langue», Le Français dans le monde, Texte dactylographié, $16 \mathrm{p}$. (À paraître).

BOULANGER, Jean-Claude (1989f): «A la recherche d'une norme lexicographique laurentienne», Communication présentée au Colloque sur la lexicographie bilingue, Ottawa, Université d'Ottawa, 7 et 8 novembre 1989 , Texte dactylographié, $39 \mathrm{p}$. (À paraître dans les actes).

BOULANGER, Jean-Claude (1989g): «Faut-il dégriffer ou "regriffer" le dictionnaire?», Communication présentée au colloque sur l'aménagement de la langue au Québec, Auberge Mont-Gabriel, Mont-Rolland (Québec), 7 et 8 décembre 1989, Texte dactylographié, $18 \mathrm{p}$. (À paraître dans les actes).

BOULANGER, Jean-Claude (1990) : «La terminologie et le dictionnaire de langue», Conférence prononcée à la Faculteit der Letteren, Vrije Universiteit, Amsterdam, mars, Texte dactylographié, $21 \mathrm{p}$.

BOUVEROT, Danielle (1986): «Termes techniques et indicateurs de domaines dans le Suplément du Dictionnaire critique de Féraud», Autour de Féraud. La lexicographie en France de 1762 à 1835 , Actes du Colloque international organisé à l'École normale supérieure de jeunes filles, 7 au 9 décembre 1984, coll. «École normale supérieure de jeunes filles», 29, Paris, École normale supérieure de jeunes filles, pp. 157161.

CANDEL, Danielle (1979): «La présentation par domaines des emplois scientifiques et techniques dans quelques dictionnaires de langue», Langue française, 43 , septembre, pp. 100-115. [Dictionnaire, sémantique et culture]

CANDEL, Danielle (1983) : «Réflexions sur l'utilisation de textes scientifiques dans un dictionnaire de langue», Études de linguistique appliquée, Nouvelle série, 51, juillet-septembre, pp. 21-33. [Les discours scientifiques]

CAYER, Micheline (1982): «Les niveaux de langue en terminologie», Travaux de terminologie et de linguistique, 1, coll. «Études, recherches et documentation», Québec, Office de la langue française, pp. 9-20.

CORBIN, Pierre (1980): "Niveaux de langue : pèlerinage chez un archêtype», Bulletin du Centre d"analyse du discours, 4, Université de Lille III, Lille, Presses universitaires de Lille, pp. 325-353. [Synonymies]

CORBIN, Danielle et Pierre CORBIN (1980): «Le monde étrange des dictionnaires (1): les «marques d'usage», dans le Micro-Robert», Bulletin du Centre d'analyse du discours, 4, Université de Lille III, Lille, Presses universitaires de Lille, pp. 237-324. [Synonymies].

GIRARDIN, Chantal (1987): «Système des marques et connotations sociales dans quelques dictionnaires culturels français», Lexicographica, 3, pp. 76-102.

HANDWERKER, Brigitte (1989): «Savoir lexical, savoir d'expert: problèmes de représentation», DRLAV. Revue de linguistique, 40, pp. 63-87. [Signes et sens] 
L'HOMME, Marie-Claude (1990): «Existe-t-il une langue de spécialité? Points de vue pratique et théorique», Communication présentée aux Journées de linguistique $1990,1^{\text {er }}$ et 2 mars, Québec, Département de langues et linguistique, Université Laval, mars, Texte dactylographié, 14 p. (Å paraître dans les actes).

LOFFLER-LAURIAN, Anne-Marie (1982): «ÊEtre dans quelques textes de physique et de chimie hautement spécialisés», Revue de linguistique romane, 181-182, janvier-juin, pp. 121-157.

LOFFLER-LAURIAN, Anne-Marie (1983): «Faire el ses quasi-synonymes dans les discours scientifiques», Études de linguistique appliquée, Nouvelle série, 51, juillet-septembre, pp. 91-103. [Les discours scientifiques]

POIRIER, Claude (1988): «Présentation du dictionnaire», Dictionnaire du français plus. À l'usage des francophones d'Amérique, Édition établie sous la responsabilité de A.E. Shiaty, avec la collaboration de Pierre Auger et de Normand Beauchemin, Rédacteur principal : Claude Poirier, Montréal, Centre éducatif et culturel inc., pp. XIII-XIX.

QUEMADA, Bernard (1989): «Dictionnaire», Encyclopaedia Universalis, 7, Paris, Encyclopaedia Universalis, pp. 387-390.

REY, Alain (1985): «Préface de la deuxième édition», Le Grand Robert de la langue française. Dictionnaire alphabétique et analogique de la langue française, $2^{\mathrm{e}}$ édition entièrement revue et augmentée par $\mathrm{A}$. Rey, Paris, Dictionnaires Le Robert, vol. 1, pp. XVII-XLII.

REY, Alain (1989a): «Présentation du dictionnaire», Le Petit Robert. Dictionnaire alphabétique et analogique de la langue française, Nouvelle édition revue, corrigée et mise à jour, Rédaction dirigée par Alain Rey et Josette Rey-Debove, Paris, Dictionnaires Le Robert, pp. IX-XIX.

REY, Alain (1989b): "Révolution». Histoire d'un mot, coll. «Bibliothèque des histoires», Paris, Éditions Gallimard, $382 \mathrm{p}$.

REY-DEBOVE, Josette (1980): «Du bon usage du jargon», Le Bulletin du Groupe de recherches sémiolinguistiques, 13, mars, pp. 31-41.

REY-DEBOVE, Josette (1989): «Prototypes et définitions», DRLAV. Revue de linguistique, 41, pp. 143-167. [Écriture et formalismes]

\section{Dictionnaires}

ABENAIM, Raymonde, BOULANGER, Jean-Claude, SHIATY, A E et Denis VAUGEOIS (1986): Dictionnaire CEC jeunesse, Nouvelle édition, Montréal, Centre éducatif et culturel inc., 1200 p. [DCECJ]

Dictionnaire du français plus. À l'usage des francophones d'Amérique (1988), Édition établie sous la responsabilité de A.E. Shiaty, avec la collaboration de Pierre Auger et de Normand Beauchemin, Rédacteur principal : Claude Poirier, Montréal, Centre éducatif et culturel inc., XXIV + 1857 p. [DFP]

Dictionnaire Hachette de la langue française (1980), Sous la direction de Françoise Guérard, Paris, Encyclopédies Hachette, 1817 p. [DHLF]

DUBOIS, Jean, GIACOMO, Mathée, GUESPIN, Louis, MARCELLESI, Christiane, MARCELLESI, JeanBaptiste et Jean-Pierre MÉVEL (1973): Dictionnaire de linguistique, Paris, Librairie Larousse, XL + 518 p. [DdL]

GALISSON, Robert et Daniel COSTE (1976)(Sous la direction de): Dictionnaire de didactique des langues, coll. «F», Paris, Librairie Hachette, 612 p. [DDL]

Larousse de la langue française. Lexis (1979), (Sous la direction de Jean Dubois), $2^{\mathrm{e}}$ édition, illustrée, Paris, Librairie Larousse, XVI + 2111 p. [Lexis]

Lexis. Dictionnaire de la langue française (1975), (Sous la direction de Jean Dubois), Paris, Librairie Larousse, LXXIX + 1951 p. [Lexis]

Petit Larousse illustré 1975 (1972), Paris, Librairie Larousse, 1800 p. + XVI + Atlas. [PLI]

Petit Larousse illustré 1990 (1989), Paris, Librairie Larousse, I 680 p. + Atlas. [PLI]

ROBERT, Paul (1951-1964): Dictionnaire alphabétique et analogique de la langue françuise. (Le Robert), Paris, Société du Nouveau Littré - Le Robert, 6 vol., p. v. [GRLF]

ROBERT, Paul (1967): Le Petit Robert. Dictionnaire alphabétique et analogique de la langue française. Secrétaire général de rédaction: Alain Rey, Paris, Sociêté du Nouveau Littré, XXXII + 1972 p. [PR]

ROBERT, Paul (1985): Le Grand Robert de la langue française. Dictionnaire alphabétique et analogique de la langue française, $2^{\mathrm{e}}$ édition entièrement revue et augmentée par A. Rey, Paris, Dictionnaires Le Robert, 9 vol., LVIII p. + p. v. [GRLF]

ROBERT, Paul (1989): Le Petit Robert 1. Dictionnaire alphabétique et analogique de la langue française, Nouvelle édition revue, corrigée et mise à jour, Rédaction dirigée par Alain Rey et Josette Rey-Debove, Paris, Dictionnaires Le Robert, XXXI + 2175 p. [PR] 